$\mathbb{T}$ periodica polytechnica

\author{
Civil Engineering \\ 58/4 (2014) 387 396 \\ doi: 10.3311/PPci.7084 \\ http://periodicapolytechnica.org/ci \\ Creative Commons Attribution (1) \\ RESEARCH ARTICLE \\ Karoly A. Zalka \\ Received 2013-10-14, accepted 2014-07-07
}

\section{Maximum deflection of asymmetric wall-frame buildings under horizontal load}

\begin{abstract}
A new analytical procedure is presented for the determination of the maximum deflection of asymmetric multi-storey buildings braced by frameworks, shear walls and cores. The complex response of the building is separated into two phenomena: lateral deflection and rotation. A closed-form solution is given for the torsional problem leading to a relatively simple calculation. The solution is obtained using an analogy between the bending and torsion of structural systems. The accuracy of the proposed method is demonstrated using the results of over one hundred test structures of different bracing system arrangements, different stiffness characteristics and different heights ranging from four storeys to eighty storeys. Step-by-step instructions and a practical example worked out to the smallest detail are presented to aid practical application.
\end{abstract}

\section{Keywords \\ deflection $\cdot$ torsion $\cdot$ continuum method $\cdot$ asymmetric $\cdot$ three- dimensional behaviour}

Karoly A. Zalka

Visiting Professor, Budapest University of Technology and Economics, Múegyetem rkp. 3, H-1111 Budapest, Hungary

e-mail: zalkak@t-online.hu

\section{Introduction}

The maximum deflection of a multi-storey building is a vital piece of information as its magnitude should always be restricted in one way or another. Unfortunately, its determination represents a formidable task as the three-dimensional behaviour of the building leads to a complex problem where a great number of stiffness and geometrical characteristics are involved. Interaction among the bracing units occurs and, mainly due to their different type of deformation, they have an effect on one another. In addition, in the general case when the bracing system is not doubly symmetric, lateral movements are combined with rotation.

The area of the lateral problem is very well researched and documented and a great number of methods - too many to list here - have been made available for the handling of the pure sway problem. However, the situation is markedly different when lateral deflection is accompanied by rotation, i.e., when the building has an asymmetric bracing system arrangement. The saying "There is no such thing as a twist-free building" is well known in the structural engineering community, both in practice and among researchers; still, due to the complexity of the three-dimensional behaviour, the torsional problem has not been thoroughly investigated, let alone solved in a comprehensive manner. Considerable efforts have been made regarding the torsional behaviour of individual structural elements [3, 10] but the global torsional behaviour of whole structural systems is a less cultivated area. Even the widely used treasure house of structural engineering research [12] only deals with symmetric wall-frame buildings that do not twist. There are some excellent publications that offer relatively simple solution for the global torsional problem [2, 3, 5, 7, 9, 11] but they are either still too complicated or of limited applicability and none of them is backed up with a comprehensive accuracy analysis. All the above shortcomings were addressed in a recent paper [15] which offered a closed-form solution for the maximum rotation of regular multi-storey buildings. However, that solution is still fairly complicated and, as it will be shown in this paper, its accuracy can significantly be improved.

To handle this three-dimensional problem in a simple way 
seems to be a hopeless task using conventional tools. However, by relying on an analogy between bending and torsion, a relatively simple solution can be produced. The aim of this paper is threefold: (a) to establish a new model for the analysis using this analogy, (b) to produce a simple closed-form solution for the rotation of a building, that clearly shows the contribution of the different stiffness characteristics to the torsional resistance and (c) to show how the proposed method can be used for the determination of the maximum deflection of multi-storey asymmetric building structures.

Although large frameworks and even whole buildings are now routinely analysed using computer packages, the proposed method may be useful from several aspects. It helps the structural engineer to understand the complex three-dimensional behaviour and thus enables him/her to manipulate the stiffnesses and the location of the bracing units in such a way that optimum structural arrangement is achieved. The proposed method may also prove to be useful at the preliminary design stage when quick checks are needed with different structural arrangements. Its usefulness cannot be overemphasized for checking the results of a finite element (computer-based) analysis when the input procedure may involve tens of thousands of data and mishandling one datum may have catastrophic consequences.

The continuum method will be used and it will be assumed for the analysis that the structures under uniformly distributed horizontal load are

- regular in the sense that their characteristics do not vary over the height

- at least four storeys high with identical storey heights

- sway structures with built-in lower end at ground floor level and free upper end

and that

- the floor slabs have great in-plane and small out-of-plane stiffness

- the deformations are small and the material of the structures is linearly elastic

- the torsional stiffnesses of the individual bracing cores are negligible (compared to the global torsional stiffnesses of the bracing system).

\section{Three-dimensional behaviour. The solution of the planar problem}

When a multi-storey wall-frame building of asymmetric bracing system arrangement is subjected to horizontal load, the structure responds in a complex manner and develops both lateral displacements and rotation over the height of the building. The two phenomena can be separated, making it possible to deal with the deflection and rotation problems independently of each other. The procedure is demonstrated in Fig. 1 where the resultant of the horizontal load (per unit height) is represented by $w=w^{*} L$. Force $w$ passing through centroid $C$ is transferred to shear centre $O$ where it is accompanied by torque $m=w x_{c}$. Force $w$ in the shear centre only causes uniform lateral displacements while torque $m$ only develops rotations around the shear centre axis enabling the separate treatment of the two phenomena. Accordingly, the deflection at any location is expressed by

$$
v=v_{o}+v_{\varphi}
$$

where $v_{o}$ is the uniform part of the deflection caused by force $w$ in the shear centre and $v_{\varphi}$ is the other part of the deflection cased by torque $m=w x_{c}$.

The maximum deflection of the building develops at the top at one of the corner points of the plan of the building:

$$
v_{\max }=v(H)=v_{o}(H)+x_{\max } \varphi(H)=v_{o}(H)+\left(L-\bar{x}_{o}\right) \varphi(H)
$$

where $H$ is the height of the building, $x_{\max }$ is the distance of the corner point (where the maximum deflection occurs) from the shear centre and $\varphi(H)$ is the angle of rotation.

Let's deal with the planar problem first. The case when the resultant of the external load passes through the shear centre the planar problem - is discussed in detail in [16]. The maximum deflection of such a system of frameworks, shear walls and cores can be obtained using the $i$ th unit of the system:

$$
v_{o}(H)=\frac{q_{i} w H^{4}}{8 E I_{f, i}}+\frac{q_{i} w H^{2}}{2 K_{i} s_{i}^{2}}-\frac{q_{i} w E I_{i}}{K_{i}^{2} s_{i}^{3}}\left(\frac{1+\kappa_{i} H \sinh \kappa_{i} H}{\cosh \kappa_{i} H}-1\right)
$$

The three terms in Eq. (3) clearly identify three phenomena: the deflection is characterized by the bending and shear modes (as defined by the first and second terms) and their interaction (third term). The interaction is always beneficial as it always reduces the deflection.

Two possibilities are differentiated in using Eq. (3): (a) the system of $f$ frameworks and $m$ shear walls/cores is considered as it is (the "simple" method) or (b) the system is first reduced to $f$ frameworks by incorporating the $m$ stiffnesses of the shear walls/cores into the original frameworks creating $f$ new frameworks (the "more accurate" method).

In both cases the calculation is based on the three characteristic stiffnesses of the $i$ th framework. Term $K_{i}$ represents the shear stiffness:

$$
K_{i}=\left(\frac{1}{K_{c, i}}+\frac{1}{K_{b, i}}\right)^{-1}=K_{b, i} \frac{K_{c, i}}{K_{b, i}+K_{c, i}}=K_{b, i} r_{i}
$$

with $r_{i}$ being a modifier:

$$
r_{i}=\frac{K_{c, i}}{K_{b, i}+K_{c, i}}
$$

The shear stiffness has two "components"; $K_{b, i}$ is related to the beams while $K_{c, i}$ is linked to the columns of the framework. 


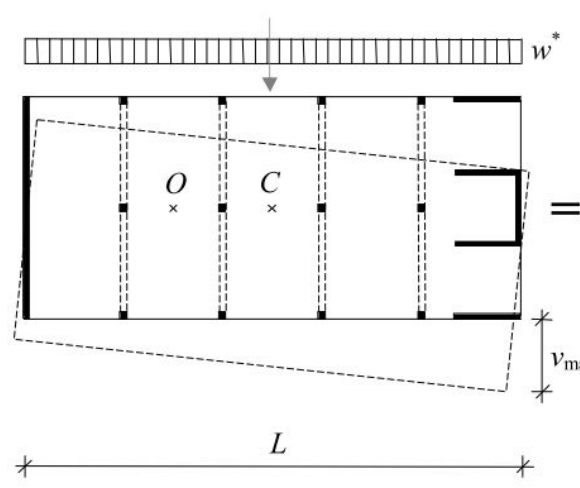

a) original problem

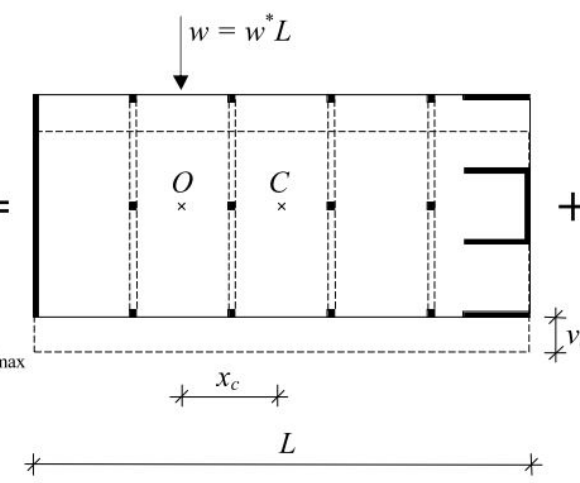

b) lateral deflection caused by $w$

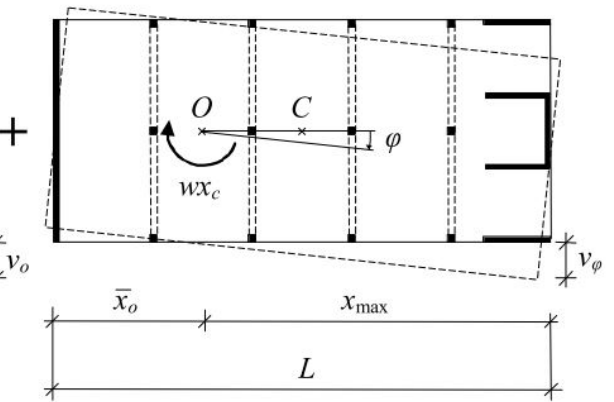

c) rotation caused by $m=w x_{c}$

Fig. 1. Asymmetric building under horizontal load.

They are defined as

$$
\begin{aligned}
K_{b, i}= & \frac{12 E I_{b, i}}{l h} \\
& \text { and } \\
K_{c, i}= & \frac{12 E I_{c, i}}{h^{2}}
\end{aligned}
$$

where $E$ is the modulus of elasticity, $h$ is the storey height and $l$ is the bay. Second moments of area $I_{b, i}$ and $I_{c, i}$ are the sums of the second moments of area of the beams and columns, respectively, of the $i$ th framework.

The local bending stiffness of the $i$ th framework is defined as

$$
E I_{i}=E I_{c, i} r_{i}
$$

where modifier $r_{i}$ is used to avoid the overrepresentation of the bending stiffness of the columns [4,14].

The global bending stiffness of the $i$ th framework is given by

$$
E I_{g, i}=E \sum_{j=1}^{n} A_{c, j} t_{j}^{2}
$$

where $A_{c, j}$ is the cross-sectional area of the $j$ th column of the $i$ th framework, $t_{j}$ is its distance from the centroid of the crosssections and $n$ is the number of columns.

The sum of the local and global bending stiffnesses represents the total bending stiffness of the framework:

$$
E I_{f, i}=E I_{i}+E I_{g, i}
$$

Eq. (3) also contains some auxiliary quantities:

$$
\begin{aligned}
\kappa_{i} & =\sqrt{a_{i}+b_{i}}, \\
a_{i} & =\frac{K_{i}}{E I_{g, i}}, \\
b_{i} & =\frac{K_{i}}{E I_{i}}, \\
s_{i} & =1+\frac{a_{i}}{b_{i}}=1+\frac{I_{i}}{I_{g, i}}
\end{aligned}
$$

Finally, apportioner $q_{i}$ defines the load share on the $i$ th bracing unit. Its value is determined using the "governing" stiffnesses of the bracing units. The "governing" stiffness of the $i$ th bracing unit is defined as the reciprocal of the maximum (in-plane) deflection of the unit in question:

$$
S_{i}=\frac{1}{v_{i}(H)}
$$

When the "governing" stiffnesses are available, the apportioner is calculated as

$$
q_{i}=\frac{S_{i}}{\sum_{i=1}^{f} S_{i}}
$$

Eq. (3) can be used for the determination of the maximum deflections $v_{i}(H)$. For this purpose, an arbitrary value of apportioner $q_{i}$, say $q_{i}=1$, can be used, as its value eventually drops out of the calculations.

To ensure best accuracy, when the apportioners - and load shares - are determined, the co-called "more accurate" method should be used - see [16] for detailed explanation. It is only mentioned here that according to the "more accurate" method, the problem of $f+m$ frameworks and shear walls/cores is reduced to the problem $f$ frameworks. This is done by incorporating the stiffnesses of the $m$ shear walls and cores into the original $f$ frameworks, according to the "governing" stiffnesses of the original frameworks. This procedure results in $f$ new frameworks as reflected by Eq. (12) where summation goes from 1 to $f$.

The torsional problem - whose solution $\varphi$ is also needed in Eq. (2) - will be discussed in detail in the next section.

\section{The torsional problem}

Two totally different approaches can be considered for handling the torsional problem. A complete procedure can be followed from scratch by examining the equilibrium of the bracing system. This leads to a very lengthy procedure - as is demonstrated by the derivation related to a bracing system consisting of shear walls and cores only [14]. The other possibility is the application of the well-known analogy in the stress analysis of 
thin-walled structures in bending and torsion [8, 13]. This avenue is followed here. According to the analogy, translations, bending moments and shear forces correspond to rotation, warping moments and torsional moments, respectively.

The first step in applying the bending-torsion analogy is the establishment of the corresponding stiffnesses. The characteristic stiffnesses with the bending analysis are the local bending stiffness, the global bending stiffness and the shear stiffness given by Eqs. (7), (8) and (4), respectively. The corresponding stiffnesses for the torsional analysis are as follows.

Stiffness $E I$ is the local bending stiffness with the deflection analysis. The corresponding stiffness with the torsional analysis is the local warping torsional stiffness:

$$
E I_{\omega}=E I t^{2}
$$

where $t$ is the perpendicular distance of the bracing unit in question from the shear centre Fig. 2

Stiffness $E I_{g}$ is the global bending stiffness with the deflection analysis. The corresponding stiffness with the torsional analysis is the global warping torsional stiffness:

$$
E I_{g \omega}=E I_{g} t^{2}
$$

The total warping torsional stiffness is the sum of the local and global torsional stiffnesses:

$$
E I_{f \omega}=E I_{\omega}+E I_{g \omega}=E\left(I+I_{g}\right) t^{2}=E I_{f} t^{2}
$$

Stiffness $K$ is the shear stiffness with the deflection analysis. The corresponding stiffness with the torsional analysis is the Saint-Venant torsional stiffness:

$$
(G J)=K t^{2}
$$

In addition to stiffnesses Eqs. (13), (14) and (16), the individual bracing units - especially the cores - may have their "own" warping and Saint-Venant torsional stiffnesses but they are normally small and, in accordance with the basic assumption made in the Introduction, they are neglected here.

Before the analogy is fully utilised, the location of the shear centre has to be established as it is needed for the determination of the above torsional stiffnesses. The shear centre is defined as the centre of the "governing" stiffnesses of the bracing units. The "governing" stiffness of each bracing unit is given by Eq. (11) where the maximum deflection is needed. It is obtained using Eq. (3). In the case of a shear wall or a core, Eq. (3) reduces to its first term.

With the "governing" stiffnesses of the bracing units, the calculation of the location of the shear centre is best carried out in the co-ordinate system $\bar{x}-\bar{y}$, whose origin lies in the upper left corner of the plan of the building and whose axes are aligned with the sides of the building Fig. 2 ,

$$
\begin{gathered}
\bar{x}_{o}=\frac{\sum_{1}^{f+m} S_{y, i} \bar{x}_{i}}{\sum_{1}^{f+m} S_{y, i}} \\
\text { and } \\
\bar{y}_{o}=\frac{\sum_{1}^{f+m} S_{x, i} \bar{y}_{i}}{\sum_{1}^{f+m} S_{x, i}}
\end{gathered}
$$

In the above formulae

$\bar{x}_{i}, \bar{y}_{i}$ are the perpendicular distances of the $i$ th bracing unit from $\bar{y}$ and $\bar{x}$

$f \quad$ is the number of frameworks

$m \quad$ is the number of shear walls / cores

$S_{x, i}$

$S_{y, i} \quad$ are the "governing" stiffnesses by Eq. 11 in direction $x$ and $y$

\section{The solution of the torsional problem}

All the stiffnesses and other geometrical characteristics are now available for the application of the analogy. Looking at the derivations of the lateral (sway) problem [16], two possibilities may be considered. With the sway problem, two procedures were developed: the "simple" method and the "more accurate" method. As the term suggests, the "simple" method offers a simple solution while the "more accurate" method results in a more accurate albeit more complicated solution. Careful investigation of the two procedures automatically answers the question "Which procedure to adopt?". The more accurate method was developed by incorporating the stiffnesses of the shear walls and cores into the frameworks. This approach makes it possible to take into consideration the effect of interaction more accurately than the other method, leading to a more accurate solution. With the torsional problem, however, another phenomenon enters the picture. The location of the shear centre plays a very important role and becomes part of the geometrical characteristics. When the stiffnesses of the shear walls and cores are incorporated into the frameworks, the procedure indeed leads to a more accurate handling of the effect of interaction between the shear and bending modes but, at the same time, somewhat distorts the behaviour, as far as the location of the shear centre of the bracing system is concerned. This follows from the fact that by removing some of the bracing units from their original places and creating new, "virtual" frameworks (by incorporating the shear walls and cores into the frameworks), the location of the shear centre of the bracing system is altered. This would be unacceptable with the torsional problem. (As the location of the shear centre was irrelevant with the planar problem, it was possible to make use of the advantage of the "more accurate" method without its detrimental effect.)

It follows that the "simple" method should be applied and the analysis must centre on the original system of $f$ frameworks and $m$ shear walls/cores when the analogy is used for the solution 


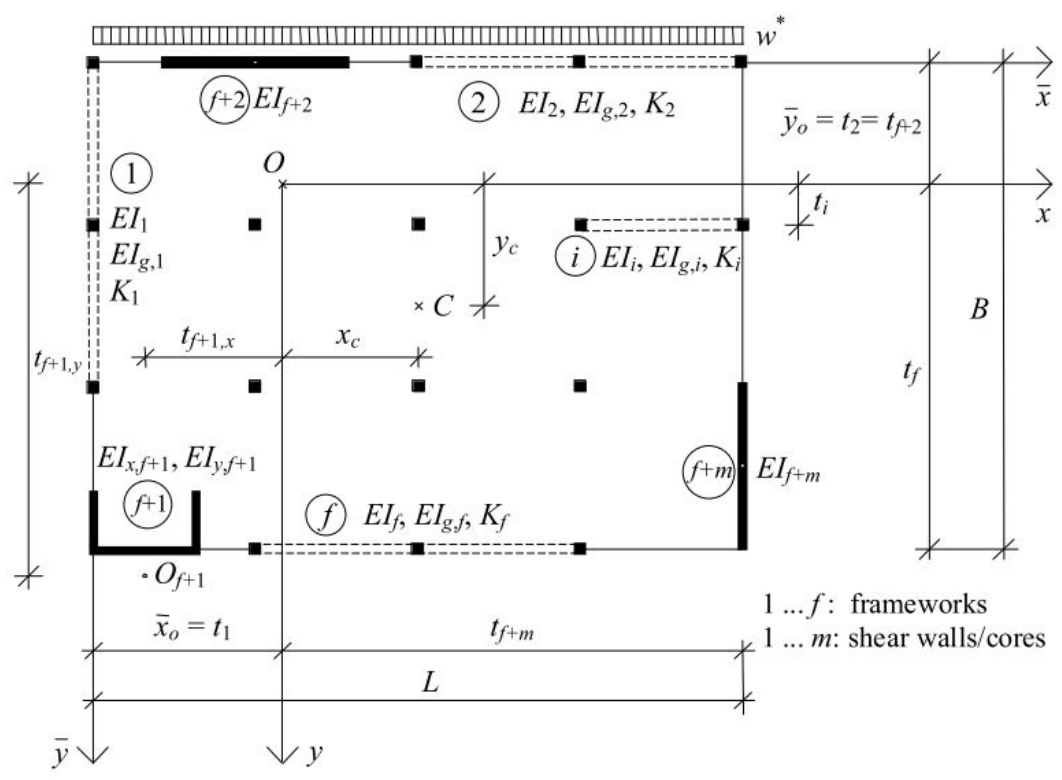

Fig. 2. Bracing system for the torsional analysis.

of the torsional problem. In doing so, and using the torsional stiffnesses introduced by Eqs. (13), (14), (15) and (16), the governing differential equation of the torsional problem is obtained as

$$
\varphi^{\prime \prime \prime \prime}-\kappa_{i}^{2} \varphi^{\prime \prime}=\frac{q_{\omega, i} m}{E I_{\omega, i}}\left(\frac{a_{i} z^{2}}{2}-1\right)
$$

where subscript $i$ refers to the $i$ th bracing unit. Auxiliary quantities $\kappa_{i}$ and $a_{i}$ are identical to those used in the planar problem, i.e., they are given by Eq. 10]. This follows from the fact that when the step-by-step requirements of the analogy are met and the corresponding stiffnesses are matched, moment arms $t_{i}$ drop out of the formulae. Eq. (18) also contains the total torsional moment (per unit height) on the bracing system:

$$
m=w x_{c}=w\left(\frac{L}{2}-\bar{x}_{o}\right)
$$

Torsional apportioner $q_{\omega, i}$ in Eq. (18) plays the same role as $q_{i}$ in the planar case. Its value is obtained using the "governing" torsional stiffnesses of the bracing units. The "governing" torsional stiffness of the $i$ th bracing unit is defined as

$$
S_{\omega, i}=S_{i} t_{i}^{2}=\frac{t_{i}^{2}}{v_{i}(H)}
$$

where $v_{i}(H)$ is the maximum deflection of the $i$ th bracing unit.

The torsional apportioner can now be determined:

$$
q_{\omega, i}=\frac{S_{\omega, i}}{\sum_{1}^{f+m} S_{\omega, i}}
$$

Note that summation goes from 1 to $f+m$.

The torsional moment share on the $i$ th bracing unit is

$$
m_{i}=q_{\omega, i} m
$$

Finally, in completing the application of the analogy, the formula for the rotation emerges as

$$
\begin{aligned}
& \varphi_{i}(z)=\frac{m_{i}}{E I_{f \omega, i}}\left(\frac{H^{3} z}{6}-\frac{z^{4}}{24}\right)+\frac{m_{i} z^{2}}{2(G J) s_{i}^{2}}- \\
& -\frac{m_{i} E I_{\omega, i}}{(G J)^{2} s_{i}^{3}}\left(\frac{\cosh \kappa_{i}(H-z)+\kappa_{i} H \sinh \kappa_{i} z}{\cosh \kappa_{i} H}-1\right)
\end{aligned}
$$

Bearing in mind that the above rotation calculated using the characteristics of the $i$ th bracing unit is identical to the rotation of the building and that maximum rotation develops at $z=H$, the formula for the maximum rotation emerges as

$$
\begin{aligned}
& \varphi_{\max }=\varphi_{i}(H)= \\
& \frac{m_{i} H^{4}}{8 E I_{f \omega, i}}+\frac{m_{i} H^{2}}{2(G J) s_{i}^{2}}-\frac{m_{i} E I_{\omega, i}}{(G J)^{2} s_{i}^{3}}\left(\frac{1+\kappa_{i} H \sinh \kappa_{i} H}{\cosh \kappa_{i} H}-1\right)
\end{aligned}
$$

Auxiliary quantity $s_{i}$ is given in Eq. (10).

Naturally, Eq. (24) is identical to Eq. (3) in structure. The torsional mode is characterized by the warping and Saint-Venant torsional modes and the resulting rotation is reduced by the effect of the interaction between the two modes. The interaction is always beneficial. Eq. (24) (together with Eqs. (13), (14), 15) and 16 also demonstrate that the rotations of the building can be reduced by increasing the bending and shear stiffnesses of the bracing units and, perhaps more importantly, by increasing the effective (perpendicular) distances of the bracing units from the shear centre. However, the most efficient way of reducing rotations (to zero) is to minimise the external torque (to zero) by eliminating the arm of the wind load, in other words, by creating a bracing system where the resultant of the wind load passes through the shear centre. 


\section{Practical application: worked example}

When the formula for the maximum deflection was developed above, the presentation followed an order that was most suitable for, and in line with, the theoretical considerations. For practical applications, however, it is advisable to follow a different order to simplify and minimize the amount of calculation.

The procedure is best carried out in four steps.

1 The basic stiffness characteristics, the maximum deflection, the "governing" stiffness (and, if needed, the apportioner) for each bracing unit are calculated $\left(E I, E I_{g}, K, v_{\max }, S, q\right)$.

2 The maximum deflection of the shear centre axis is determined after incorporating the stiffnesses of the shear walls and cores into the frameworks in the relevant direction \{Eq. (3)\}.

3 Having determined the location of the shear centre and then the torsional stiffnesses of the bracing units, the maximum rotation of the system is determined \{Eq. 24]\}.

4 The maximum deflection of the building is obtained by adding up its two components \{Eq. [2\}\}.

This procedure is demonstrated below using a 28-storey building whose layout is shown in Fig. 3 The building is subjected to a uniformly distributed horizontal load of intensity $w *=1 \mathrm{kN} / \mathrm{m}^{2}$.

The bracing system consists of four frameworks, three shear walls and a U-core. The storey-height is $h=3 \mathrm{~m}$ and the total height of the building is $H=28 \times 3=84 \mathrm{~m}$. The modulus of elasticity is $E=25 \times 10^{6} \mathrm{kN} / \mathrm{m}^{2}$. The cross-sectional characteristics of the bracing units are given in Table 1 . The stiffness of the shear walls perpendicular to their plane is ignored.

PART 1: The basic characteristics of the bracing units

Framework F7 (Bracing units 1, 2, 3 and 4)

With the part shear stiffnesses given by Eq. (6)

$$
\begin{aligned}
& K_{b, 1}=\frac{12 E I_{b}}{l h}=\frac{12 \cdot 25 \cdot 10^{6} \cdot 0.0042 \dot{6}}{6 \cdot 3}=71111 \mathrm{kN} \\
& K_{c, 1}=\frac{12 E I_{c}}{h^{2}}=\frac{12 \cdot 25 \cdot 10^{6} \cdot 0.0064}{3^{2}}=213333 \mathrm{kN}
\end{aligned}
$$

the shear stiffness of the framework is calculated using Eq. (4)

$$
\begin{aligned}
& K_{1}=K_{b, 1} \frac{K_{c, 1}}{K_{b, 1}+K_{c, 1}}=K_{b, 1} r_{1}= \\
& =71111 \frac{213333}{71111+213333}=71111 \cdot 0.75=53333 \mathrm{kN}
\end{aligned}
$$

which also furnishes the value of modifier $r_{1}=0.75$.

The local bending stiffness is given by Eq. 77:

$$
\begin{aligned}
& E I_{1}=E I_{c, 1} r_{1}= \\
& 25 \cdot 10^{6} \cdot 0.0064 \cdot 0.75=120000 \mathrm{kNm}^{2}
\end{aligned}
$$

The global bending stiffness is calculated using Eq. (8):

$$
\begin{aligned}
& E I_{g, 1}=E \sum_{j=1}^{n} A_{j} t_{j}^{2}= \\
& 25 \cdot 10^{6} \cdot 0.4 \cdot 0.4 \cdot 6^{2} \cdot 2=288000000 \mathrm{kNm}^{2}
\end{aligned}
$$

The sum of the local and global stiffnesses [Eq. 99] is

$$
E I_{f, 1}=E I_{1}+E I_{g, 1}=288120000 \mathrm{kNm}^{2}
$$

With auxiliary quantities $a_{1}, b_{1}, s_{1}$ and $\kappa_{1}$ obtained from Eq. 10] as

$$
\begin{aligned}
& a_{1}=\frac{K_{1}}{E I_{g, 1}}=\frac{53333}{288000000}=0.000185, \\
& b_{1}=\frac{K_{1}}{E I_{1}}=\frac{53333}{120000}=0.44444 \\
& s_{1}=1+\frac{a_{1}}{b_{1}}=1+\frac{0.000185}{0.44444}=1.000416, \\
& \kappa_{1}=\sqrt{0.000185+0.44444}=0.6668, \\
& \kappa_{1} H=56.0
\end{aligned}
$$

the maximum deflection of the framework is calculated using Eq. (3) (with $q_{1}=1$ ):

$$
\begin{aligned}
v_{1}= & \frac{30 \cdot 84^{4}}{8 \cdot 288120000}+\frac{30 \cdot 84^{2}}{2 \cdot 53333 \cdot 1.000416^{2}}- \\
& -\frac{30 \cdot 120000}{53333^{2} \cdot 1.000416^{3}}\left(\frac{1+56 \sinh 56}{\cosh 56}-1\right)= \\
= & 0.648+1.983-0.070=2.561 \mathrm{~m}
\end{aligned}
$$

The governing stiffness of the framework is given by Eq. 11:

$$
\begin{gathered}
S_{1}=\frac{1}{v_{1}(H)}=\frac{1}{2.561}=0.39 \mathrm{~m}^{-1} \\
\text { As } v_{2}=v_{3}=v_{4}=v_{1}=2.561 \mathrm{~m} \text { holds, } \\
S_{2}=S_{3}=S_{4}=0.39 \mathrm{~m}^{-1}
\end{gathered}
$$

Shear wall W5 (Bracing Unit 5)

The maximum (in-plane) deflection and the stiffness of shear wall W5 are obtained using the first term in Eq. (3) (with $q_{5}=1$ ) and Eq. 11], respectively, as

$$
\begin{aligned}
& v_{5}=\frac{w H^{4}}{8 E I_{5}}=\frac{30 \cdot 84^{4}}{8 \cdot 25 \cdot 10^{6} \cdot 43.2}=0.1729 \mathrm{~m}, \\
& S_{5}=\frac{1}{v_{5}(H)}=\frac{1}{0.1729}=5.784 \mathrm{~m}^{-1}
\end{aligned}
$$

$U$-core (Bracing Unit 6) \{Only $I_{x, 6}$ and deflection in plane $z y$ are relevant\}

The maximum deflection and the stiffness of the core are calculated using the first term of Eq. (3) (with $q_{6}=1$ ) and Eq. (11), respectively: 
Tab. 1. Cross-sectional characteristics of the bracing units.

\begin{tabular}{cccccc}
\hline Bracing unit & $\begin{array}{c}\text { cross-section of } \\
\text { columns }\end{array}$ & $\begin{array}{c}\text { cross-section of } \\
\text { beams }\end{array}$ & $I_{c, i}\left[\mathrm{~m}^{4}\right]$ & $I_{b, i}\left[\mathrm{~m}^{4}\right]$ & $I_{g, i}\left[\mathrm{~m}^{4}\right]$ \\
\hline F7 & $0.4 \times 0.4$ & $0.4 \times 0.4$ & 0.0064 & 0.00426 & 11.52 \\
W2 & $0.2 \times 4.0$ & - & 1.066 & - & - \\
W5 & $0.3 \times 12.0$ & - & 43.2 & - & - \\
U & $h=b=4.0, t=0.3, e=1.714$ & $I_{x}=11.245$ & - & - \\
\hline
\end{tabular}

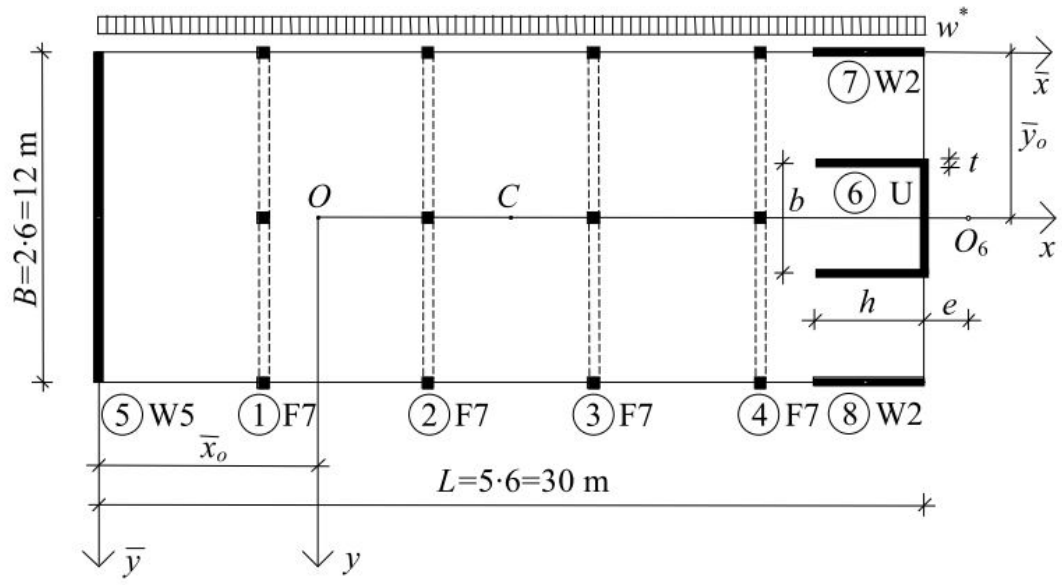

Fig. 3. Layout for the worked example.

$$
\begin{aligned}
& v_{6}=\frac{w H^{4}}{8 E I_{x, 6}}=\frac{30 \cdot 84^{4}}{8 \cdot 25 \cdot 10^{6} \cdot 11.245}=0.664 \mathrm{~m}, \\
& S_{6}=\frac{1}{v_{6}(H)}=\frac{1}{0.664}=1.506 \mathrm{~m}^{-1}
\end{aligned}
$$

Shear wall W2 (Bracing Units 7 and 8)

The maximum (in-plane) deflection and the stiffness of shear wall W2 are obtained using the first term in Eq. (3) (with $\left.q_{7}=1\right)$ and Eq. (11), respectively, as

$$
\begin{aligned}
& v_{7}=v_{8}=\frac{w H^{4}}{8 E I_{7}}=\frac{30 \cdot 84^{4}}{8 \cdot 25 \cdot 10^{6} \cdot 1.0 \dot{6}}=7.0 \mathrm{~m} \\
& S_{7}=S_{8}=\frac{1}{v_{7}(H)}=\frac{1}{7.0}=0.143 \mathrm{~m}^{-1}
\end{aligned}
$$

PART 2: The maximum deflection of the shear centre axis

The participating bracing units are the four frameworks $(1,2$, $3,4)$, shear wall 5 (5) and the U-core (6). There is no need for the calculation of load shares as the four frameworks are identical. It is sufficient to consider one framework only which takes one fourth of the external load. It is also sufficient to consider one framework (when the shear wall and core are incorporated into the frameworks) which takes one fourth of the bending stiffnesses of the shear wall and the core. The local bending stiffness of this new framework, say $F 7^{*}$, is

$$
\begin{aligned}
& E I_{1}^{*}=E\left(I_{1}+\frac{1}{4}\left(I_{5}+I_{6 x}\right)\right)= \\
& =120000+25 \cdot 10^{6} \frac{1}{4}(43.2+11.245)=340.4 \cdot 10^{6} \mathrm{kNm}^{2}
\end{aligned}
$$

The global bending stiffness and the shear stiffness are unchanged at

$$
\begin{aligned}
E I_{g, 1}= & 288000000 \mathrm{kNm}^{2} \\
& \text { and } \\
K_{1}= & 53333 \mathrm{kN}
\end{aligned}
$$

and the total bending stiffness is

$$
E I_{f, 1}^{*}=E I_{1}^{*}+E I_{g, 1}=(340.6+288) 10^{6}=628.4 \cdot 10^{6} \mathrm{kNm}^{2}
$$

With the new auxiliary quantities ( $a_{1}$ is unchanged)

$$
\begin{aligned}
& b_{1}^{*}=\frac{K_{1}}{E I_{1}^{*}}=\frac{53333}{340.4 \cdot 10^{6}}=0.000157, \\
& s_{1}^{*}=1+\frac{a_{1}}{b_{1}^{*}}=1+\frac{0.000185}{0.000157}=2.178 \\
& \kappa_{1}^{*}=\sqrt{a_{1}+b_{1}^{*}}=\sqrt{0.000185+0.000157}=0.0185
\end{aligned}
$$

and

$$
\kappa_{1}^{*} H=1.553
$$

the maximum deflection of the shear centre axis is obtained using Eq. (3) (with $q_{1}=1 / 4$ ):

$$
\begin{aligned}
& v_{o}=\frac{0.25 \cdot 30 \cdot 84^{4}}{8 \cdot 628.4 \cdot 10^{6}}+\frac{0.25 \cdot 30 \cdot 84^{2}}{2 \cdot 53333 \cdot 2.178^{2}}- \\
& -\frac{0.25 \cdot 30 \cdot 340.4 \cdot 10^{6}}{53333^{2} \cdot 2.178^{3}}\left(\frac{1+1.553 \sinh 1.553}{\cosh 1.553}-1\right)=0.107 \mathrm{~m}
\end{aligned}
$$


PART 3: Maximum rotation around the shear centre

The participating bracing units are the four frameworks (F7), the three shear walls (W5, W2 and W2) and the U-core. The U-core is only "active" in plane $y z$ (with $I_{x, 6}$ ) as the length of its other (perpendicular) moment arm is zero $\left(t_{6 y}=0\right)$.

The location of the shear centre and then the torsional stiffnesses of the bracing units are needed first. Because of symmetry, only one of the two co-ordinates needs calculation. Based on Eq. (17), the shear centre co-ordinates are

$$
\begin{aligned}
& \bar{x}_{o}=\frac{\sum_{1}^{f+m} S_{y, i} \bar{x}_{i}}{\sum_{1}^{f+m} S_{y, i}}=\frac{S_{1}(6+12+18+24)+S_{6}(L+e)}{S_{5}+4 S_{1}+S_{6}}= \\
& =\frac{0.39 \cdot 60+1.506 \cdot 31.714}{5.784+4 \cdot 0.39+1.506}=8.04 \mathrm{~m}, \\
& \bar{y}_{o}=6 \mathrm{~m}
\end{aligned}
$$

The torsional moment causing rotation around the shear centre is given by Eq. (19):

$$
m=w\left(\frac{L}{2}-\bar{x}_{o}\right)=30(15-8.04)=208.8 \mathrm{kNm} / \mathrm{m}
$$

The "governing" torsional stiffnesses of the bracing units are obtained from Eq. 20, using their perpendicular distance from the shear centre and their maximum deflection:

$$
\begin{aligned}
& S_{\omega, 1}=\frac{t_{1}^{2}}{v_{1}}=\frac{2.04^{2}}{2.561}=1.625 \mathrm{~m}, \\
& S_{\omega, 2}=\frac{t_{2}^{2}}{v_{2}}=\frac{3.96^{2}}{2.561}=6.123 \mathrm{~m}, \\
& S_{\omega, 3}=\frac{t_{3}^{2}}{v_{3}}=\frac{9.96^{2}}{2.561}=38.73 \mathrm{~m}, \\
& S_{\omega, 4}=\frac{t_{4}^{2}}{v_{4}}=\frac{15.96^{2}}{2.561}=99.46 \mathrm{~m}, \\
& S_{\omega, 5}=\frac{t_{5}^{2}}{v_{5}}=\frac{8.04^{2}}{0.1729}=373.87 \mathrm{~m}, \\
& S_{\omega, 6}=\frac{t_{6}^{2}}{v_{6}}=\frac{23.674^{2}}{0.6641}=843.94 \mathrm{~m}, \\
& S_{\omega, 7}=S_{\omega, 8}=\frac{t_{7}^{2}}{v_{7}}=\frac{6^{2}}{7.0}=5.143 \mathrm{~m}
\end{aligned}
$$

With the sum of the torsional stiffnesses

$$
\sum_{1}^{f+m} S_{\omega, i}=1374.0 \mathrm{~m}
$$

Eq. 21] can now be used to determine the torsional load share on one of the bracing units. Choosing, say, Bracing Unit 5, the torsional load share is

$$
q_{\omega, 5}=\frac{S_{\omega, 5}}{\sum_{1}^{f+m} S_{\omega, i}}=\frac{373.87}{1374.0}=0.2721
$$

The maximum rotation of the building can now be determined using Eq. (22) and Eq. (24) which, because Bracing Unit 5 is a shear wall, reduces to its first term (and $I_{f \omega}$ reduces to $I_{\omega}$ ):

$$
\begin{aligned}
& \varphi_{\max }=\varphi_{5}(H)=\frac{q_{\omega, 5} m H^{4}}{8 E I_{\omega, 5}}= \\
& =\frac{0.2721 \cdot 208.8 \cdot 84^{4}}{8 \cdot 25 \cdot 10^{6} \cdot 43.2 \cdot 8.04^{2}}=0.005065 \mathrm{rad}
\end{aligned}
$$

PART 4: The maximum deflection of the building

Maximum deflection develops at the right-hand side of the building where, according to Eq. (2), the two components of the deflection add up:

$$
\begin{aligned}
& v_{\max }=v_{o}(H)+\left(L-\bar{x}_{o}\right) \varphi(H)= \\
& =0.107+0.005065(30-8.04)=0.218 \mathrm{~m}
\end{aligned}
$$

The Finite Element based computer program Axis (2003) gives $v_{\max }=0.208 \mathrm{~m}$ as the maximum deflection of the building.

\section{Accuracy analysis}

The result of the worked example offers some indication regarding the accuracy of the proposed method but, clearly, more information is needed if the proposed procedure is to be used for practical application. In order to carry out a comprehensive accuracy analysis, in addition to the worked example above, twelve more bracing system arrangements were created (Fig. 4) using frameworks, shear walls and cores.

These individual bracing units (frameworks F1, F2, F3, F4, F5, F6 and F7, shear walls W2, W3, W4 and W5 and the Ucore) were all used for the accuracy analysis of the planar solution [16]. The cross-sections of the columns and beams of the frameworks were $0.4 / 0.4$ (metre) unless otherwise indicated in Fig. 4. The storey height and the bays were 3 metres and 6 metres, respectively, in each case. The height of the structures varied from 4 storeys to 80 storeys in nine steps. This resulted in 117 test structures. The bracing units and systems as well as the layouts were chosen in such a way as to cover a wide range of structures. Among the bracing systems, there are bending dominated systems, shear dominated systems, mixed systems, systems dominated by frameworks, systems dominated by shear wall, systems developing dominant lateral deflection, systems very vulnerable to rotations, etc. The modulus of elasticity for the concrete structures was $25 \mathrm{kN} / \mathrm{mm}^{2}$.

The Finite Element based computer program Axis (2003) was used for the determination of the maximum deflection of the bracing systems and these results were considered "exact".

The error of the proposed method was defined as the difference between the "exact" and approximate results, related to the "exact" solution. Positive error meant conservative estimates. Table 2 offers a summary regarding the performance of the proposed method giving the range of error, the average absolute error and the maximum error. The maximum deflections were also determined using the "old" method [15]. The comparison shows that the proposed method is much superior concerning the average absolute error and the maximum error. What the table 


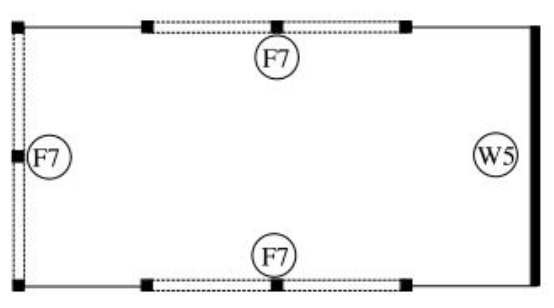

a) $\mathrm{F} 7+\mathrm{F} 7+\mathrm{F} 7+\mathrm{W} 5$

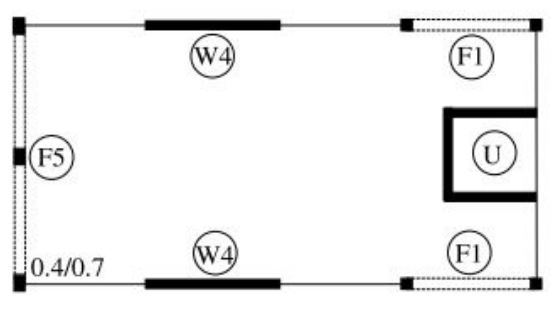

d) $\mathrm{F} 1+\mathrm{F} 5+\mathrm{F} 1+\mathrm{U}+\mathrm{W} 4+\mathrm{W} 4$

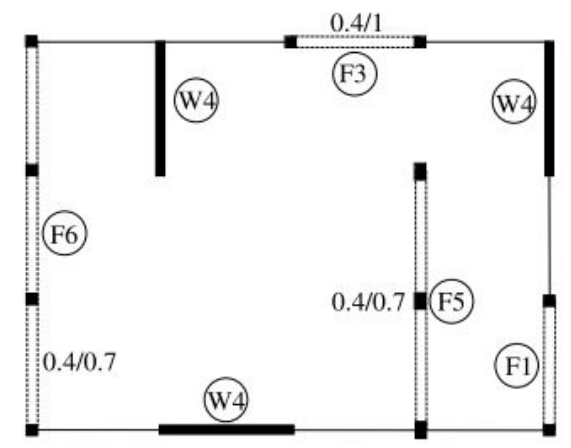

g) $\mathrm{F} 1+\mathrm{F} 3+\mathrm{F} 5+\mathrm{F} 6+\mathrm{W} 4+\mathrm{W} 4+\mathrm{W} 4$

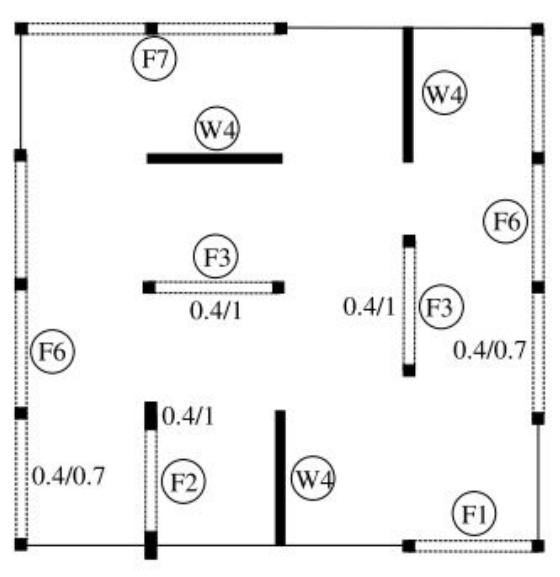

j) $\mathrm{F} 1+\mathrm{F} 2+\mathrm{F} 3+\mathrm{F} 3+\mathrm{F} 6+\mathrm{F} 6+\mathrm{F} 7+\mathrm{W} 4+\mathrm{W} 4+\mathrm{W} 4$

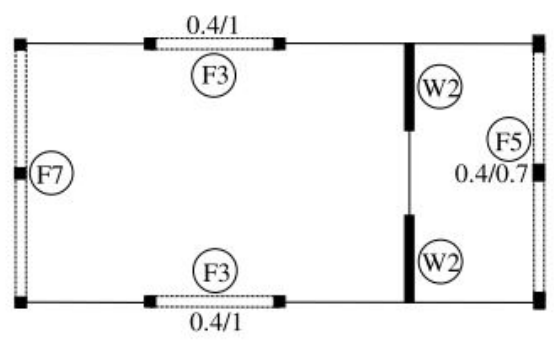

b) $\mathrm{F} 3+\mathrm{F} 3+\mathrm{F} 5+\mathrm{F} 7+\mathrm{W} 2+\mathrm{W} 2$

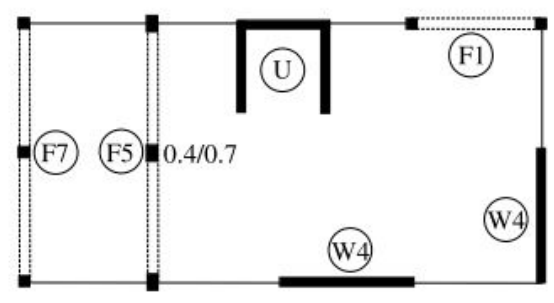

e) $\mathrm{F} 1+\mathrm{F} 5+\mathrm{F} 7+\mathrm{U}+\mathrm{W} 4+\mathrm{W} 4$

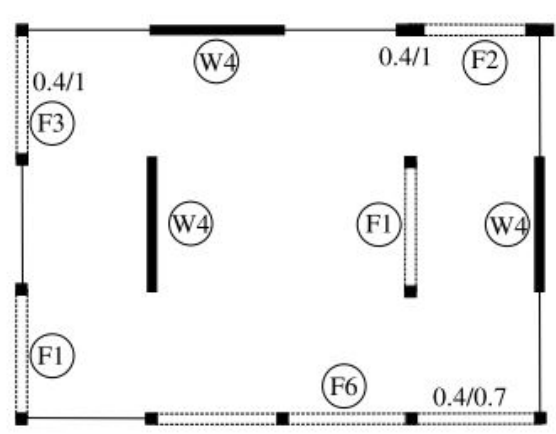

h) $\mathrm{F} 1+\mathrm{F} 1+\mathrm{F} 2+\mathrm{F} 3+\mathrm{F} 6+\mathrm{W} 4+\mathrm{W} 4+\mathrm{W} 4$

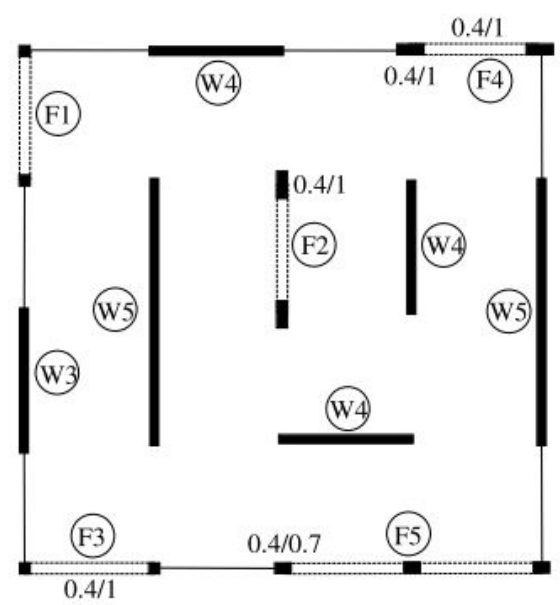

k) $\mathrm{F} 1+\mathrm{F} 2+\mathrm{F} 3+\mathrm{F} 4+\mathrm{F} 5+\mathrm{W} 3+\mathrm{W} 4+\mathrm{W} 4+\mathrm{W} 4+\mathrm{W} 5+\mathrm{W} 5$

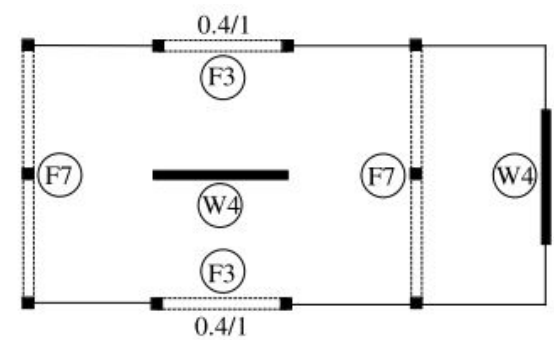

c) $\mathrm{F} 3+\mathrm{F} 3+\mathrm{F} 7+\mathrm{F} 7+\mathrm{W} 4+\mathrm{W} 4$

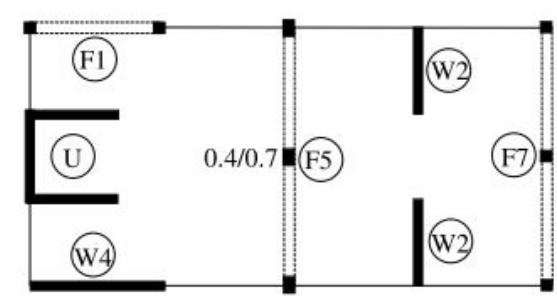

f) $\mathrm{F} 1+\mathrm{F} 5+\mathrm{F} 7+\mathrm{U}+\mathrm{W} 2+\mathrm{W} 2+\mathrm{W} 4$

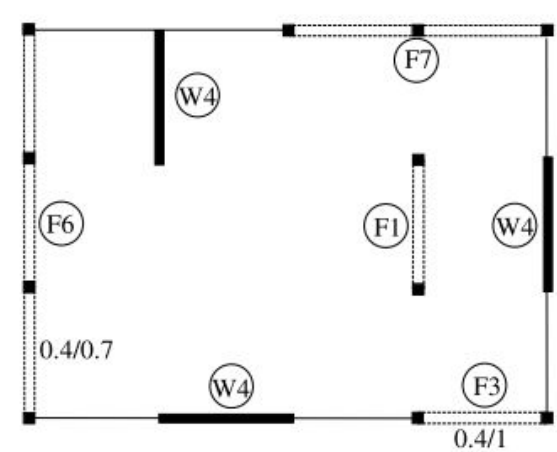

i) $\mathrm{F} 1+\mathrm{F} 3+\mathrm{F} 6+\mathrm{F} 7+\mathrm{W} 4+\mathrm{W} 4+\mathrm{W} 4$

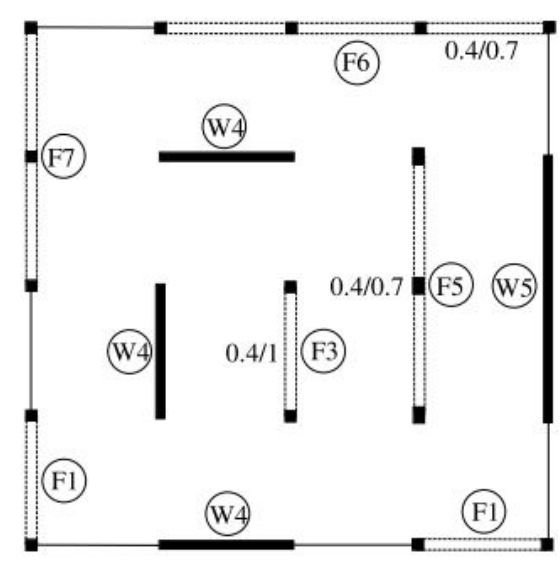

1) $\mathrm{F} 1+\mathrm{F} 1+\mathrm{F} 3+\mathrm{F} 5+\mathrm{F} 6+\mathrm{F} 7+\mathrm{W} 4+\mathrm{W} 4+\mathrm{W} 4+\mathrm{W} 5$

Fig. 4. Structures for the accuracy analysis.

Tab. 2. Accuracy of the "old" and proposed methods.

\begin{tabular}{cccc}
\hline Method & Range of error (\%) & $\begin{array}{c}\text { Average absolute error } \\
(\%)\end{array}$ & Maximum error (\%) \\
\hline "Old" method 15 & 1 to 23 & 9 & 23 \\
\hline Proposed method & -7 to 15 & 5 & 15 \\
\hline
\end{tabular}


does not show is the ease of use and in this respect the proposed procedure also outperforms the old one by a large margin.

The performance of the proposed method over the height of the building is demonstrated in Fig. 5 .

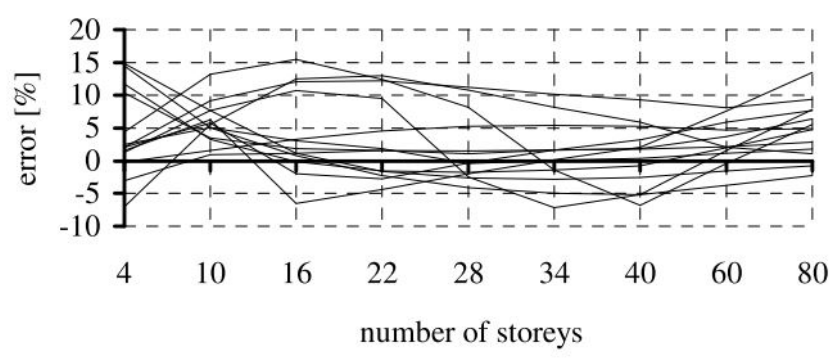

Fig. 5. Accuracy of the proposed method over the height.

The results of the 117 test cases also demonstrated that the beneficial effect of the interaction between the bending and shear (warping and Saint-Venant torsional) modes may be significant in the case of low-rise buildings but as the height of the structures increases this effect becomes rapidly negligible.

\section{Conclusions}

The application of the continuum method and the analogy between the bending and torsion of bars make it possible to carry out the torsional analysis of regular multi-storey buildings in a simple manner. The resulting closed-form solution for the maximum rotation of the building offers a clear picture. The torsional behaviour is defined by three distinctive phenomenon: warping torsion, Saint-Venant torsion and the interaction between the two modes. In identifying the key contributors to the torsional resistance, the efficiency of the bracing system can easily be maximised. The interaction between the two modes is always beneficial. However, this interaction - that may be significant for low-rise buildings - rapidly becomes negligible as the height of the structure increases. The formula for the maximum rotation is identical in structure to the formula of the maximum deflection of the shear centre axis and they together lead to the determination of the maximum deflection of asymmetric multistorey buildings in a single and simple step.

As for the accuracy of the proposed method, a comprehensive accuracy analysis of 117 test structures resulted in an error range of $7 \%$ to $+15 \%$, with a less than $5 \%$ absolute average error. Knowing the uncertainties in building materials and inaccuracies in the construction industry, the proposed method is considered accurate enough for either preliminary design or for checking purposes.

\section{References}

1 AXIS VM, Finite Element Program for Structural Analysis. Version 7. User's Manual, InterCAD Kft., 2003.

2 Coull A, Wahab AFA, Lateral load distribution in asymmetrical tall building structures, Journal of Structural Engineering, ASCE, 119, (1993), pp. 1032-1047.
3 Council on Tall Buildings, Planning and Design of Tall Buildings, American Society of Civil Engineers; New York, 1978. a Monograph in 5 volumes.

4 Hegedús I, Kollár LP, Application of the sandwich theory in the stability analysis of structures, In: Kollár $\mathbf{L}$ (ed.), Structural stability in engineering practice, E \& FN Spon; London, 1999, pp. 187-241.

5 Hoenderkamp HJCD, Approximate deflection analysis of non-symmetric high-rise structures, In: Beedle LS (ed.), Habitat and the high-rise - Tradition and innovation, Proceedings of the Fifth World Congress, Council on Tall Buildings and Urban Habitat; Lehigh University, Bethlehem, USA, May 14-19, 1995, pp. 1185-1209.

6 Howson WP, Rafezy B, Torsional analysis of asymmetric proportional building structures using substitute plane frames, In: Proceedings of the 3rd International Conference on Advances in Steel Structures, Vol. II, Elsevier; Hong Kong, 2002, pp. 1177-1184.

7 Irwin AW, Design of shear wall buildings, Construction Industry Research and Information Association; London, 1984. Report 102.

8 Kollbunner CF, Basler K, Torsion in Structures, Springer-Verlag; Berlin, New York, 1969, DOI 10.1007/978-3-662-22557-8

9 Nadjai A, Johnson D, Torsion in tall buildings by a discrete force method, The Structural Design of Tall Buildings, 7(3), (1998), pp. 217-231.

10 Seaburg PA, Carter CJ, Torsional analysis of structural steel members (Design Guide 9), American Institute of Steel Construction, 2003.

11 Schueller W, The vertical building structure, Van Nostrand Reinhold; New York, 1990.

12 Stafford Smith B, Coull A, Tall building structures. Analysis and design, John Wiley \& Sons; New York, 1991.

13 Vlasov VZ, Thin-walled elastic beams, 2nd edn, Israeli Program for Scientific Translations; Jerusalem, 1961. Moscow, 1959.

14 Zalka KA, Armer GST, Stability of large structures, ButterworthHeinemann; Oxford, 1992.

15 Zalka KA, Torsional analysis of multi-storey building structures under horizontal load, The Structural Design of Tall and Special Buildings, 22(3), (2013), pp. 126-143, DOI 10.1002/tal.665

16 Zalka KA, Maximum deflection of symmetric wall-frame buildings, Periodica Polytechnica, Civil Engineering, 57(2), (2013), pp. 173-184, DOI 10.3311/PPci.7172 